\title{
Knowledge and practice regarding the behavioural risks of cancer among college students in Ethiopia
}

\author{
Foziya Mohammed Hussien ${ }^{1}$, Anissa Mohammed Hassen ${ }^{1}$, Zinet Abegaz Asfaw ${ }^{1}$, Aragaw Yimer Ahmed $^{2}$, Yeshimebet Ali Dawed ${ }^{1}$, \\ Ahmed Hussien Asfaw ${ }^{1}$, Erkihun Tadesse Amsalu ${ }^{1}$ and Hamid Yimam Hassen ${ }^{3}$
}

${ }^{1}$ Department of Public Health, College of Medicine and Health Science, Wollo University, Dessie 1145, Ethiopia

${ }^{2}$ Department of Internal Medicine, School of Medicine, College of Medicine and Health Science, Wollo University, Dessie 1145, Ethiopia

${ }^{3}$ Department of Public Health, College of Medicine and Health Science, Mizan-Tepi University, Mizan Teferi 260, Ethiopia

\begin{abstract}
In recent years, morbidity and mortality due to cancer has been increasing in Ethiopia, putting it among the most important public health issues. Cancer and associated complications can be prevented provided that effective interventions are put in place to control risk factors. Therefore, this study aimed to assess the level of knowledge and practice of preventable behavioural risk factors of cancer. We conducted a cross-sectional study among 200 college students in Northeast Ethiopia utilising quantitative methods of data collection. Data on socioeconomic characteristics, health belief variables, knowledge and behavioural risk factors (alcohol consumption, tobacco smoking, physical activity and dietary practice) were collected. The level of knowledge and practice was summarised using descriptive statistics. To investigate the variation in knowledge and practice across sociodemographic characteristics, we performed Pearson Chi-square test or Fisher's exact test. The majority (81.0\%) of participants was male and $82.0 \%$ were in the age group of $18-24$ years. More than half $(61.0 \%)$ of them had poor knowledge about the behavioural risk factors of cancer. Nearly one-third (30.5\%) consume alcohol, whereas $16.0 \%, 18.0 \%$ and $20.0 \%$, respectively, smoke tobacco, consume street food and packedfried snacks daily. Alcohol consumption $(p=0.02)$ and level of vigorous physical activity $(p=0.001)$ were significantly higher among males than females. Alcohol consumption, tobacco smoking and unhealthy dietary practice were remarkably high, whereas knowledge towards the behavioural risk factors was low. Therefore, health education and collaborative action between different sectors are needed to counter the emerging problem.
\end{abstract}

The trial is registered in ClinicalTrials.gov (https://register.clinicaltrials.gov) NCT04269018.

Keywords: knowledge, behavioural risk factor, cancer, student, Ethiopia

\section{Introduction}

Improvement in global health has improved life expectancy, enhanced maternal and child health and reduced the spread and consequence of infectious diseases [1]. However, major challenges to human health and development persist, with chronic diseases including cancer still the major cause of death and disability [2, 3]. By the year 2040, the global
Correspondence to: Foziya Mohammed Hussien Email: foziyamohammed2018@gmail.com

ecancer 2022, 16:1343

https://doi.org/10.3332/ecancer.2022.1343

Published: $13 / 01 / 2022$

Received: 05/08/2021

Publication costs for this article were supported by ecancer (UK Charity number 1176307).

Copyright: $@$ the authors; licensee ecancermedicalscience. This is an Open Access article distributed under the terms of the Creative Commons Attribution License (http:// creativecommons.org/licenses/by/4.0), which permits unrestricted use, distribution, and reproduction in any medium, provided the original work is properly cited. 
cancer burden is expected to exceed 27 million new cancer cases per year [4]. Globally, with an estimated 19.3 million cancer incident cases and 10 million deaths in 2020, it is the second leading cause of death next to cardiovascular diseases [5, 6]. Previously, Non-Communicable Diseases (NCDs) including cancer were considered a disease of high-income countries, but now they are also an important public health issue in low- and middle-income countries (LMICs) [6, 7]. Lifestyle changes, rapid urbanisation, cultural transition and an increase in life expectancy could contribute to a rise in incidence $[6,8]$. This imposes an enormous burden on the already overwhelmed healthcare system of LMICs since cancer treatment centres are not sufficient to cover the increasing needs of the affected population [9,10].

Despite a steady increase in cancer morbidity and mortality, it has been largely neglected by the governments of LMICs, international organisations and civil societies due to the existing infectious diseases. As a result, we should not forget the terrible effect on human lives and economic cost which is imposed by cancer [9]. Additionally, poor surveillance, research and unresponsive health policies remain a challenge to the prevention of cancer in LMICs [11].

In Ethiopia, the numbers of cancer cases were estimated to be 77,352 and were responsible for 51,865 deaths nationally in 2020 [12]. Cancers of the breast (20.9\%), cervix (9.6\%), colorectal (7.8\%), leukaemia (5.6\%) and Non-Hodgkin lymphoma (4.9\%) are the most commonly occurring cancers in the country [12]. In Ethiopia, public health policy gives little attention to cancer prevention and control. Recently, the Federal Ministry of Health launched the National Cancer Control Programme focusing on the multi-sectoral integration of cancer prevention and control [13]. However, the rise in cancer incidence has emphasised that the control practices are still suboptimal.

Risky behaviours including alcohol consumption and cigarette smoking are higher among college students than the general population [14-18]. School-based intervention programmes and policy setting on substance use and other risky behaviours are important to reduce the modifiable risk factors of cancer [17]. Therefore, studies at various levels and contexts are important to insight policy makers on the potential area of interventions.

In Ethiopia, cancer screening, diagnosis and management are sub-optimal and population-based data are limited to few cities [20]. Few studies in the country showed poor knowledge towards risk factors and screening, which were identified as important factors for poor utilisation of cancer screening and other prevention services [21-23]. Thus, investigating the knowledge, perception and practice of the modifiable risk factors of cancers is crucial to develop and implement targeted preventive interventions to halt the growing burden of cancer.

\section{Materials and methods}

\section{Study setting, design and sampling}

A cross-sectional study was conducted to assess knowledge, perception and practice towards the behavioural risk factors of cancer among college students in Dessie, Northeast Ethiopia in July 2021. Dessie town is $401 \mathrm{kms}$ far from Addis Ababa, the capital city of Ethiopia. The commonly cultivated staple foods in the study area are barley, oats, bean and teff. There are three public colleges in the city, which provide several academic and training services. All public colleges in the city were included in the study, namely Woizero Siheen Polytechnic College, Dessie College of Teachers Education and Dessie Health Science College. Woizero Siheen Polytechnic College was established in 1930 and currently provides technical and vocational education and training in five different campuses: Main Campus (Siheen Campus), Merho Campus, Hotie Campus, Dawdo Campus and Menen (Vocational Campus). The College has 5,975 trainees in regular and night-shift programmes. The college is also providing short-term, non-formal and in-company training. Dessie College of Teachers Education was established in 1980 and has 1,426 students.

This study is a baseline assessment of a randomised control trial aimed to evaluate the effectiveness of mobile text messages on knowledge and perception towards cancer and behavioural risks among college students in Northeast Ethiopia. The trial was registered at ClinicalTrials.gov (https://register.clinicaltrials.gov) NCT04269018 and the protocol was published in PLOS ONE [24]. The sample size was determined using superiority trial design and then a total of 200 participants were enrolled in the study. The sample size was allocated to Woizero Siheen Polytechnic College and Dessie College of Teachers Education based on proportional to the number of students. Students' identification (ID) was obtained from the registrar's office of each college and used as a sampling frame. Then, a simple random sampling method was used to select 
eligible study subjects. Students in the selected public colleges aged 18-35 years with no prior diagnosis of any type of cancer were included, whereas students of Health Science College were excluded as they are expected to know the risks of cancer and preventive mechanisms.

\section{Data collection and quality control}

We captured data using self-administered questionnaires on sociodemographic, knowledge, perception and practice of the behavioural risks of cancer. Knowledge was assessed with 19 questions regarding high-risk groups, risk factors, complications and preventive mechanisms of cancer with 'yes', 'no' and 'I do not know' options. The correct responses were assigned a score of 1, whereas 'No' or 'I do not know' responses were assigned 0 [25]. The cumulative mean score of knowledge of students was estimated. As a result, those who had scored less than the mean were considered to have poor knowledge and those who had scored greater than or equal to the mean value were considered as having good knowledge [26].

To assess the risk perception of cancer, we obtained data on the health belief model components: Perceived susceptibility towards cancer (e.g. You perceived that you will get risk of developing cancer at any time of your life), Perceived severity of cancer (e.g. 'Cancer is fatal, and its complication are dangerous/severe in life'), Perceived benefits of adopting behaviours (e.g. 'Having a healthy nutrition would decrease the probability of any type of cancers'), Perceived barriers to adopting behaviours (e.g. 'My monthly income is insufficient to take the recommended diet for the prevention of cancer'), Perceived self-efficacy for adopting dietary behaviours (e.g. 'I am confident that I can prevent cancer through healthy lifestyle'), Internal cues for adopting dietary behaviours (e.g. 'when knowing the death of any by cancer, it flips me to perform the preventive behaviors'), the items of this subscale were measured on a Likert scale ranging from $1=$ 'Strongly disagree' to $5=$ 'Strongly agree' [27].

Physical activity level was measured using the International Physical Activity Questionnaires short form. This contains the frequency and time participants spent doing vigorous physical activity, moderate physical activity, walking and sitting in the last 7 days. Alcohol intake was assessed using the Alcohol Use Disorders Identification Test (AUDIT), a tool adapted from the World Health Organization (WHO) which assessed participant's consumption in the past 1 month [28]. The frequency and quantity of alcohol consumption were classified into light (1 and 2 drinking occasions per week), moderate ( 3 and 4 drinking per week) and heavy ( 5 and more drinking per week) [29]. Moreover, tobacco use was measured using a series of questions adapted from the WHO, which assessed the duration, quantity and frequency of smoking. Current smokers were defined as those who smoked 100 cigarettes in their lifetime and currently smoke cigarettes, whereas ever-smokers represent those who smoked at least 100 cigarettes in their lifetime but quit smoking during the survey [30]. Smokers were classified as light (smoking 1 to 10 cigarettes sticks per day), medium (smoking 11 to 20 cigarettes per day), heavy (smoking 20-40 cigarettes per day) and very heavy smokers (smoking on average more than 40 cigarettes per day) [31]. Dietary habit was measured using a food frequency questionnaire adapted from the Dietary Assessment Primer which was developed by the USA National Cancer Institute and then modified based on the study objectives and local staple foods [32]. The type and frequency of food items scaled with Never Consumed (NC) = 1, Once Per Month $(\mathrm{OPM})=2$, Two to Three per Month $($ TTM $)=3$, Once per Week $(\mathrm{OW})=4$, Two times per Week $($ TW) $=5$, Three to Four times per Week (TFW) $=6$, Five to Six times per Week(FSW) $=7$, every day $(E D)=8$ were assessed. One month food consumption pattern with foods consumed either inside or outside the home and greater than one tablespoon were measured. We used principal component analysis (PCA) to generate diet scores and classify food consumption patterns. As a result, study participants were categorised into three groups such as poor healthy eating habit, medium healthy eating habit and good healthy eating habit.

The data were collected by newly graduated BSc nurses. After we received an official letter from Wollo University, each college's administrator and the registrar were contacted to confirm their willingness and to obtain a student list with ID number. We approached 200 students and fortunately, all of them gave their consent to participate in this study.

The validity of the items was checked by an expert panel of specialists in health education, oncology and nutrition. They provided intellectual judgment on the necessity and relevance of the scaling items. The questionnaire was first prepared in English language and translated to Amharic and again re-translated into English by another person to check for consistency. About $10 \%$ of the questionnaire was pretested then internal consistency was checked (Cronbach's alpha $=0.80$ ). To control the data quality, all data collectors were trained for 2 days on the aim of the study, methods and how to take informed consent and administer the questionnaire. Moreover, supervisors closely monitored the data collection process. 


\section{Data analysis}

Upon checking for completeness and consistency, data were coded and entered using EpiData version 3.1 (https://epidata-entry.software. informer.com/3.1/). Then, the clean data were exported to a free statistical software R version 4.0.3 for further processing and analysis. Descriptive statistics including absolute and relative frequencies, and cross-tabulations were used to summarise categorical variables. For continuous variables, the normality of the data was checked and then summarised using either mean with standard deviation (SD) or median with interquartile range (IQR). PCA was computed to generate diet score. To investigate the variation in cancer risk knowledge and behaviour across sex, we used Pearson Chi-square statistic or Fisher's exact test.

\section{Results}

\section{Sociodemographic characteristics}

The mean age of participants was 21.6 years (SD: 3.3), and 82.0\% were within the age group 18-24 years. Majorities (81.0\%) were male, $87.5 \%$ were single, $54.5 \%$ were Muslims and $89.4 \%$ were from the Amhara region. The median family size was 5.5 (IQR: $4-7)$ persons per household. The median household monthly income was 7,000 Ethiopian birr (1 ETB 0.025 USD) (IQR: 4,875-10,000) (Table 1).

Table 1. Sociodemographic characteristics of students in Dessie public colleges, $2021(N=200)$.

\begin{tabular}{|l|c|c|}
\hline \multicolumn{1}{|c|}{ Variable } & Frequency & Percent \\
\hline Sex & & \\
\hline Male & 162 & 81.0 \\
\hline Female & 38 & 19.0 \\
\hline Age group (years) & & \\
\hline 18-24 & 164 & 82.0 \\
\hline 25-30 & 31 & 15.5 \\
\hline 31-35 & 5 & 2.5 \\
\hline Religion & 109 & \\
\hline Muslim & 91 & 54.5 \\
\hline Orthodox & & 45.5 \\
\hline Ethnicity & 378 & 89.4 \\
\hline Amhara & 24 & 5.7 \\
\hline Tigre & 19 & 4.5 \\
\hline Oromo & 2 & 0.5 \\
\hline Others & 175 & 2.5 \\
\hline Marital status & & \\
\hline Never married & 3 & 1.5 \\
\hline Currently married & 15 & \\
\hline Divorced & & \\
\hline Widowed & & \\
\hline Separated & & \\
\hline & & \\
\hline
\end{tabular}




\section{Knowledge and perception towards the behavioural risk factors of cancer}

Out of a maximum of 19 , the mean knowledge score was $6.6(\mathrm{SD}=3.8$ ) and $61.0 \%$ of students had poor knowledge on the behavioural risk factors of cancer. The average score of perceived susceptibility and severity was $11.0(\mathrm{SD}=3.5)$ and $21.2(\mathrm{SD}=5.7)$, respectively. The mean score of perceived benefit was 24.4 (SD = 6.3), whereas the score for the perceived barrier was $23.36(S D=6.32)$. Similarly, the mean score of cues to action and self-efficacy was $17.9(\mathrm{SD}=5.2)$ and $28.7(\mathrm{SD}=7.6)$, respectively.

\section{Behavioural risk factors of cancer}

Thirty-two (16.0\%) students reported as current smokers, of which $93.7 \%$ smoke daily. Twenty-two (68.8\%) are light smokers and $37.5 \%$ started smoking within the age of 16-20 years. Fourteen (7.0\%) have smoked cigarettes in the past daily, but stop smoking currently. Nineteen $(9.5 \%)$ students used smokeless tobacco or someone smoked within their presence. Nearly one-third (30.5\%) of students consumed alcohol in their lifetime, of which $90.2 \%$ drank 1 month preceding the survey. Nearly one-fourth (23.6\%) were light alcohol drinkers, while $20 \%$ usually used alcohol with meals (Table 2).

Table 2. Cigarette smoking and alcohol consumption among students in Dessie public colleges, $2021(N=200)$.

\begin{tabular}{|l|c|c|}
\hline \multicolumn{1}{|c|}{ Variable } & Frequency & Percent \\
\hline Currently smoking & & \\
\hline Yes & 32 & 16.0 \\
\hline No & 168 & 84.0 \\
\hline Daily smoking & & \\
\hline Yes & 30 & 93.7 \\
\hline No & 2 & 6.3 \\
\hline Type of smoker & & \\
\hline Light & 22 & 68.8 \\
\hline Medium & 8 & 25.0 \\
\hline Heavy & 1 & 3.1 \\
\hline Very heavy & 1 & 3.1 \\
\hline Age onset of smoking & & \\
\hline Unknown & 15 & 46.9 \\
\hline 10-15 & 5 & 15.6 \\
\hline 16-20 & 12 & 37.5 \\
\hline Ever smoker & 181 & 9.5 \\
\hline Yes & & \\
\hline No & 19 & 9.0 \\
\hline Smokeless tobacco & & \\
\hline Yes & & 9.5 \\
\hline No & & \\
\hline Passive smoker & & \\
\hline Yes & & \\
\hline
\end{tabular}


Table 2. Cigarette smoking and alcohol consumption among students in Dessie public colleges, $2021(N=200)$. (Continued)

\begin{tabular}{|l|c|c|}
\hline No & 181 & 90.5 \\
\hline Ever alcohol drinking & & \\
\hline Yes & 61 & 30.5 \\
\hline No & 139 & 69.5 \\
\hline Alcohol drinking within a month & & \\
\hline Yes & 55 & 90.2 \\
\hline No & 6 & 9.7 \\
\hline Drinking frequency/week & & \\
\hline Light (<2) & 13 & 23.6 \\
\hline Moderate (2-4) & 19 & 34.6 \\
\hline Heavy (5+) & 23 & 41.8 \\
\hline Drinking with meals & & \\
\hline Usually & 11 & 20 \\
\hline Sometimes & 9 & 16.4 \\
\hline Rarely & 15 & 27.2 \\
\hline Never & 20 & 36.4 \\
\hline
\end{tabular}

One month preceding the survey, 86 students were engaged in vigorous physical activity, of which $37.2 \%$ practised frequently $(5-7$ days per week) and $8.1 \%$ stayed for a long period (5-7 hours per day) in vigorous activity. Above two-fifths (40.5\%) of them were involved in moderate physical activity, of which $45.7 \%$ performed 3-4 days per week and $85.2 \%$ stayed $1-3$ hours per day. Half (50.5\%) of them walked or used bicycles for a minimum of 10 minutes, of which $44.6 \%$ walked frequently (5-7 days per week) and $82.2 \%$ stayed for $1-3$ hours per day. Majorities (67.0\%) stay 1-3 hours per day sitting, whereas 64.8\% sleep 7-10 hours per day (Table 3).

The mean diet score was $63.6(S D=10.7)$ with a minimum value of 46.0 and the maximum value of 102 . One-third (34.0\%) of study subjects had poor healthy eating habits and $32.5 \%$ had good healthy eating habits 1 month preceding the survey. Majorities (82.5\%) consumed a cereal-based diet daily within a month prior to the survey. Nearly half (47.2\%) consumed legumes and $22.5 \%$ consumed nuts and vegetable oils daily. Twenty-one (10.5\%) and 6.5\%, respectively, consumed fruits and vegetables daily. Nineteen (9.5\%) consumed flesh or organ three to four times per week, while none of the participants consumed fish and seafood twice per week within a month prior to the study. Only $2.5 \%$ consumed eggs and $5.5 \%$ used dairy products daily. Thirty-six (18.0\%) students consumed street foods and $4.5 \%$ used sugars and sweets more than one tablespoon daily. Nearly half (48.5\%) and $20.0 \%$, respectively, consumed salt and fried snacks daily. About $7.0 \%$ of study participants used fat and palm oil, and $16.5 \%$ used coffee and tea daily within a month preceding the survey (Table 4).

\section{Sex distribution with knowledge and behavioural risk factors of cancer}

Table 5 summarises the disparities in knowledge, perception and behaviour across sex. About 100 (61.7\%) of males and $57.9 \%$ of females had poor knowledge of the behavioural risks of cancer, but the difference was not statistically significant $(p=0.66)$. Above half $(51.2 \%)$ of males and $55.3 \%$ of females had poor dietary practice a month prior to the survey with no significant sub-group difference $(p=0.65)$. Twenty-nine (17.9\%) males and $7.9 \%$ females were smokers with no sub-group difference ( $p$-value $=0.14)$. The prevalence of alcohol drinking was higher among males (30.9\%) than females (13.2\%) and the difference was statistically significant $(p=0.02)$. More males $(48.8 \%)$ were engaged in vigorous physical activity than females $(18.4 \%)$ and the difference was statistically significant $(p<0.001)$. Sixty-nine $(42.6 \%)$ males and $31.6 \%$ females were engaged in moderate physical activity. The majority (71.2\%) of males and $52.6 \%$ females spent $1-3$ hours sitting, whereas $68.4 \%$ males and $50.0 \%$ females sleep 7-10 hours per day (Table 5). 
Table 3. Physical activity of students in Dessie public colleges, $2021(N=200)$.

\begin{tabular}{|c|c|c|}
\hline & Frequency & Percent \\
\hline \multicolumn{3}{|c|}{ Vigorous physical activity } \\
\hline Yes & 86 & 43.0 \\
\hline No & 114 & 57.0 \\
\hline \multicolumn{3}{|c|}{ Vigorous activity/week } \\
\hline 1-2 days & 28 & 32.6 \\
\hline $3--4$ days & 26 & 30.2 \\
\hline 5-7 days & 32 & 37.2 \\
\hline \multicolumn{3}{|c|}{ Duration of vigorous activity/day } \\
\hline 1-2 hours & 67 & 77.9 \\
\hline 3-4 hours & 12 & 14.0 \\
\hline $5-7$ hours & 7 & 8.1 \\
\hline \multicolumn{3}{|c|}{ Moderate activity } \\
\hline Yes & 81 & 40.5 \\
\hline No & 119 & 59.5 \\
\hline \multicolumn{3}{|c|}{ Moderate activity/week } \\
\hline 1-2 days & 27 & 33.3 \\
\hline 3-4 days & 37 & 45.7 \\
\hline 5-7 days & 17 & 21.0 \\
\hline \multicolumn{3}{|c|}{ Duration of moderate activity/day } \\
\hline $1-3$ hours & 69 & 85.2 \\
\hline 4-6 hours & 5 & 6.2 \\
\hline $7-10$ hours & 7 & 8.6 \\
\hline \multicolumn{3}{|c|}{ Walking/using bicycle } \\
\hline Yes & 101 & 50.5 \\
\hline No & 99 & 49.5 \\
\hline \multicolumn{3}{|c|}{ Walking/using bicycle/week } \\
\hline 1-2 days & 30 & 29.7 \\
\hline 3-4 days & 26 & 25.7 \\
\hline 5-7 days & 45 & 44.6 \\
\hline \multicolumn{3}{|c|}{ Duration walking/bicycle/day } \\
\hline $1-3$ hours & 83 & 82.2 \\
\hline 4-6 hours & 4 & 4.0 \\
\hline $7-10$ hours & 14 & 13.8 \\
\hline \multicolumn{3}{|c|}{ Duration of sitting/day } \\
\hline 1-3 hours & 134 & 67.0 \\
\hline 4-6 hours & 34 & 17.0 \\
\hline $7-10$ hours & 8 & 4.0 \\
\hline$>10$ hours & 22 & 11.0 \\
\hline
\end{tabular}


Table 3. Physical activity of students in Dessie public colleges, $2021(N=200)$.

(Continued)

\begin{tabular}{|l|c|c|}
\hline Sleeping duration/day & & \\
\hline $1-3$ hours & 8 & 4.1 \\
\hline 4-6 hours & 48 & 24.5 \\
\hline 7-10 hours & 127 & 64.8 \\
\hline$>10$ hours & 13 & 6.6 \\
\hline
\end{tabular}

Table 4. Dietary habit of students in Dessie public colleges, $2021(N=200)$.

\begin{tabular}{|c|c|c|c|c|c|c|c|c|}
\hline Variable & $\mathrm{NC}(\%)$ & OPM (\%) & TTM (\%) & OW (\%) & TW (\%) & TFW (\%) & FSW (\%) & ED (\%) \\
\hline Cereals & - & - & - & - & $14(7.0)$ & $12(6.0)$ & $9(4.5)$ & $165(82.5)$ \\
\hline Legumes & - & - & - & $33(16.5)$ & $30(15.0)$ & $11(5.5)$ & $31(15.5)$ & 95 (47.5) \\
\hline Nuts and Vegetable oils & $29(14.5)$ & $16(8.0)$ & $29(14.5)$ & $22(11.0)$ & $13(6.5)$ & $25(12.5)$ & $21(10.5)$ & $45(22.5)$ \\
\hline Fruits & $19(9.5)$ & $35(17.5)$ & $62(31.0)$ & $36(18.0)$ & $6(3.0)$ & $15(7.5)$ & $6(3.0)$ & $21(10.5)$ \\
\hline Vegetables & $8(4.0)$ & $35(17.5)$ & $57(28.5)$ & $34(17.0)$ & $36(18.0)$ & $7(3.5)$ & $10(5.0)$ & $13(6.5)$ \\
\hline Flesh and organ meat & $23(11.5)$ & $45(22.5)$ & $51(25.5)$ & $40(20.0)$ & $11(5.5)$ & $19(9.5)$ & $3(1.5)$ & $8(4.0)$ \\
\hline Fish and sea foods & $118(59.0)$ & $55(27.5)$ & $27(13.5)$ & - & - & - & - & - \\
\hline Eggs & $34(17.0)$ & $72(36.0)$ & $33(16.5)$ & $24(12.0)$ & $22(11.0)$ & $8(4.0)$ & $2(1.0)$ & $5(2.5)$ \\
\hline Dairy products & $23(11.5)$ & $76(38.0)$ & $30(15.0)$ & $28(14.0)$ & $11(5.5)$ & $7(3.5)$ & $14(7.0)$ & $11(5.5)$ \\
\hline Street foods & $38(19.0)$ & $29(14.5)$ & $11(5.5)$ & $40(20.0)$ & $25(12.5)$ & $16(8.0)$ & $5(2.5)$ & $36(18.0)$ \\
\hline Sugars and sweets & $19(9.5)$ & $38(19.0)$ & $36(18.0)$ & $37(18.5)$ & $30(15.0)$ & $15(7.5)$ & $16(8.0)$ & $9(4.5)$ \\
\hline Salt & - & - & $28(14.0)$ & $23(11.5)$ & $21(10.5)$ & $17(8.5)$ & $14(7.0)$ & $97(48.5)$ \\
\hline Fried and pre-packed snacks & $9(4.5)$ & $14(7.0)$ & $39(19.5)$ & $35(17.5)$ & $20(10.0)$ & $19(9.5)$ & $24(12.0)$ & $40(20.0)$ \\
\hline Butter, fat and palm oil & $26(13.0)$ & $43(21.5)$ & $29(15.5)$ & $33(16.5)$ & $27(13.5)$ & $14(7.0)$ & $14(7.0)$ & $14(7.0)$ \\
\hline Coffee and tea & $6(3.0)$ & $30(15.0)$ & $31(15.5)$ & $17(8.5)$ & $36(18.0)$ & $32(16.0)$ & $15(7.5)$ & $33(16.5)$ \\
\hline
\end{tabular}

Table 5. Cross tab results of behavioural factors with sex in public colleges, Ethiopia, 2021.

\begin{tabular}{|l|c|c|c|}
\hline \multicolumn{1}{|c|}{ Variable } & Male N (\%) & Female N (\%) & $p$-value \\
\hline Age group (years) & & & \\
\hline $18-24$ & $136(84.0)$ & $28(73.7)$ & \\
\hline $25-30$ & $26(16.0)$ & $5(13.2)$ & \\
\hline $31-35$ & 0 & $5(13.2)$ & \\
\hline Knowledge & & & \\
\hline Poor & $100(61.7)$ & $22(57.9)$ & 0.66 \\
\hline Good & $62(38.3)$ & $16(42.1)$ & \\
\hline Dietary habit & & & \\
\hline Poor & $83(51.2)$ & $21(55.3)$ & 0.65 \\
\hline Good & $79(48.8)$ & $17(44.7)$ & \\
\hline Smoking & & & \\
\hline Yes & $29(17.9)$ & $3(7.9)$ & 0.14 \\
\hline No & $133(82.1)$ & $35(92.1)$ & \\
\hline
\end{tabular}


Table 5. Cross tab results of behavioural factors with sex in public colleges, Ethiopia, 2021.

(Continued)

\begin{tabular}{|l|c|c|c|}
\hline Alcohol drinking & & & \\
\hline Yes & $50(30.9)$ & $5(13.2)$ & 0.02 \\
\hline No & $112(69.1)$ & $33(86.8)$ & \\
\hline Vigorous physical activity & & & \\
\hline Yes & $79(48.8)$ & $7(18.4)$ & 0.001 \\
\hline No & $83(51.2)$ & $31(81.6)$ & \\
\hline Moderate physical activity & & & \\
\hline Yes & $69(42.6 \%)$ & $12(31.6 \%)$ & 0.21 \\
\hline No & $93(57.4 \%)$ & $26(68.4 \%)$ & \\
\hline Sitting duration/day & & & \\
\hline $1-3$ hours & $114(71.2)$ & $20(52.6)$ & \\
\hline $4-6$ hours & $24(15.0)$ & $10(26.3)$ & \\
\hline $7-10$ hours & $8(5.0)$ & 0 & \\
\hline$>10$ hours & $14(8.8)$ & $8(21.1)$ & \\
\hline Sleeping duration/day & & & \\
\hline $1-3$ hours & $5(3.2)$ & $3(7.9)$ & \\
\hline $4-6$ hours & $36(22.8)$ & $12(31.6)$ & \\
\hline $7-10$ hours & $108(68.4)$ & $19(50.0)$ & \\
\hline$>10$ hours & $9(5.7)$ & $4(10.5)$ & \\
\hline
\end{tabular}

\section{Discussion}

Cancer prevention is key to tackling the incidence, decreasing mortality and reducing the social and economic burden that is imposed by cancer. Over half of all cancers could be prevented if appropriate measures are taken on the behavioural risk factors of cancer [19]. Hence, behaviour change is possible, powerful and cost-effective in the cancer prevention continuum [33]. Therefore, this study summarises the knowledge level and prevalence of behavioural risk factors among adults which help to ease the focus of interventions.

The majority of study participants had poor knowledge on the behavioural risk factors of cancer. This finding is consistent with several studies in different parts of the world [34-36]. Awareness of the modifiable risk factors is important and could be the primary strategy to improve cancer prevention and control [38]. Moreover, this might help people understand the potential health consequences of their actions and encourage them to make behaviour changes [37]. Therefore, it is important to improve public health messages about how lifestyle risk factors impact the chances of developing cancer.

In the National Cancer Control Programme of Ethiopia, the Ministry of Education was assigned to incorporate healthy lifestyles in the curriculum of college students [13]. However, healthy lifestyles and other cancer prevention programmes have yet to be incorporated in non-healthy students. Therefore, all stakeholders should be actively involved, committed and timely coordinate activities to successfully implement the programme and reduce the national burden of cancer.

In the present study, fruit and vegetable consumption is low, which increases the risk of various diseases including cancer [39-42]. It is supported by other studies done in Ethiopia $[43,44]$. This is due to the imbalance between the price of fruits and vegetables and affordability by the community [43]. Therefore, identifying this individual could help them find ways to include more fruit and vegetables in their diets [40]. Additionally, increasing the production and access of fruits and vegetables will help to subside the price and stabilise the market.

The majority consumed street foods and packed fried snacks daily, hence unhealthy foods persuasively increased the risk of cancer [41]. This finding is supported by a study done in Addis Ababa in which adolescents preferred packed and street snacks than home-prepared food [45]. 
Food preference, poor nutritional knowledge and food safety were the possible explanations for choosing packed fried and street foods [45]. Moreover, the price of fruits, vegetables and animal sources of foods was higher than unhealthy street foods, which are easily and widely available at a cheap cost [46]. Therefore, keeping the safety of foods all over the value chain could increase the access and year-round availability of healthy foods. Additionally, taxing unhealthy foods, subsidising the cost of healthy foods and providing fixed costs to staple fruits could help to stabilise the market. Awareness creation on the risk of unhealthy foods and restricting from college compounds could help to reduce the consumption of unhealthy foods and give value to healthy foods. The food services in colleges of Ethiopia particularly Dessie city are predominantly fried or canned snacks and sugary drinks. Thus, the college administration should consider establishing rules for college catering services to serve healthier food.

We found that males used more alcohol as compared to females which is convincingly linked with a large increase in cancer $[33,41,42,47]$. It is consistent with previous studies done in Ethiopia reportedly, male sex was found to be the independent predictor of alcohol consumption $[48,49]$. In Ethiopia, the beverage industries have increased leading to a significant increase in alcoholic beverage production and mass media advertisements coupled with many promotional awards exposing males to consume much alcohol. In the Ethiopian context males are more active on social media and spend more time in the outdoors than females. Therefore, awareness creation activities for college students are important to minimise the risk of cancer.

The physical activity level in the present study was low, which increases the risk of cancer [41, 42]. Similarly, male sex was significantly associated with vigorous physical activity in line with a study done in Debre Birhan, Ethiopia [50].This might be due to a lack of femalefriendly facilities and gender role variation, hence females engaged more in routine homework activity and child caring practice which makes them less active than males [50]. However, males are more likely to be involved in work related activities such as farming, biking and digging than females. In general, low availability of physical exercise facilities and playgrounds and high subscription fee are the main barriers to physical activity in Ethiopia [50,51]. Therefore, the sport and youth office should work in collaboration with the health and women affairs office administrators for fulfilling and making facilities (gymnasium and playground) accessible to the public. Moreover, they have to make all the facilities girls friendly and empower women to be physically active.

The present study should be interpreted considering the following limitations. Firstly, we rely only on subjective assessment of most of the outcome variables including physical activity level, alcohol consumption and smoking, which might lead to underestimation of such behaviours. Nevertheless, the use of self-administered questionnaires could reduce the social desirability bias. Secondly, we didn't collect a few important variables such as nutritional status, which take a part in the behavioural risk factors of cancer. Thirdly, to assess the dietary habit, we relied on food groups instead of food items, in which specific food items could vary in nutritional value and the actual intake of nutrients was not estimated and weighted. Lastly, this study focused on college students, which might have somewhat different knowledge, attitude and behaviour in comparison with the general population. Thus, it is hardly possible to generalise to the general population of the study area. Hence, future community-based studies are recommended.

\section{Conclusion}

Overall, alcohol consumption, tobacco smoking and unhealthy dietary practice were relatively high, whereas physical activity level was low among college students in Ethiopia. Additionally, knowledge of the behavioural risk factors of cancer is low. Therefore, awareness creation of a healthy lifestyle is important to reduce the risk of cancer. Moreover, a multi-sectoral collaboration of education, health, women's affairs and sport sectors is needed for producing active and healthy students, and their combined actions could easily address the behavioural risk factors of cancer. Incorporation of healthy life styles and other cancer control measures in the curriculum of non-healthy students might help to reduce cancer burden. Furthermore, restricting unhealthy foods from family food dishes and college compounds could help to reduce the consumption of unhealthy foods and give value to healthy foods. Furthermore, increasing the production and access of healthy foods are important to maintain the year-round availability and regular consumption of healthy foods. In conclusion, policy formulation on cancer prevention and control could help to organise activities, strength multi-sectoral collaboration and encourage stakeholders to perform their duties. As mentioned above, a high magnitude of behavioural risk factors of cancer was observed, which emphasised the necessity of text message trials for the prevention of cancer in young and productive age groups. 


\section{List of abbreviations}

ED, Every day; FSW, Five to six times per week; IQR, Interquartile range; LMICs, Low- and middle-income countries; NC, Never consumed; NCD, Non-communicable disease; OW, Once per week; OPM, Once per month; TFW, Three to four times per week; TW, Two times per week; SD, Standard deviation; WHO, World Health Organization.

\section{Acknowledgments}

The authors would like to extend their thanks to Wollo University research coordinating office for providing the opportunity to carry out this research. Moreover, the authors extend their gratitude to Woizero Siheen Polytechnic College and Dessie College of Teachers Education for allowing them to conduct this study. Finally, the authors want to express their gratitude to all study participants for their willingness.

\section{Declarations}

\section{Ethical consideration}

The protocol of this study obtained ethical approval from the Institutional Review Board of Wollo University, College of Medicine and Health Sciences. Written consent was obtained from each participant after an explanation of the study purpose, description of possible risks and benefits. Honest explanation of the study purpose, description of the benefits and an offer to answer all inquiries made to the respondents. Additionally, affirmation was given that they are free to withdraw or discontinue participation without any form of prejudice. Privacy and confidentiality of collected information were ensured throughout the process; measures were taken to ensure respect, dignity and freedom of each individual participating in the study. The study was following the principles of the declaration of Helsinki.

\section{Consent for publication}

Not applicable.

\section{Availability of data and materials}

Available on request.

\section{Competing interests}

The authors declare that they have no competing interests.

\section{Funding}

Partial funding for data collection only was obtained from Wollo University.

\section{Author contributions}

FMH designed the study, collected the data and wrote the original draft. HYH contributed to data analysis and interpretation, validation and write-up of the manuscript. Other authors contributed to data interpretation, resource acquisition, reviewing and editing. All authors read, critically revised and approved the final manuscript. 


\section{References}

1. Okamoto J and Leischow SJ (2019) Global cancer prevention, in Fundamentals of Cancer Prevention (Springer) pp 353-375

2. Wild CP (2019) The global cancer burden: necessity is the mother of prevention Nat Rev Cancer 19(3) 123-124 https://doi.org/10.1038/ s41568-019-0110-3 PMID: 30683893

3. Nagai $\mathrm{H}$ and Kim YH (2017) Cancer prevention from the perspective of global cancer burden patterns J Thorac Dis 9(3) 448 https://doi. org/10.21037/jtd.2017.02.75 PMID: 28449441 PMCID: 5394024

4. Facts G (2018) The Global Cancer Burden \& Cancer Prevention Strategies

5. Society AC (2018) Global Cancer Facts \& Figures 4th edn

6. IARC (2020) GLOBOCAN 2020: New Global Cancer Data

7. Cazap E, Magrath I, and Kingham TP, et al (2016) Structural barriers to diagnosis and treatment of cancer in low-and middle-income countries: the urgent need for scaling up J Clin Oncol 34(1) 14 https://doi.org/10.1200/JCO.2015.61.9189 PMCID: 4871996

8. Boyle P, Ngoma T, and Sullivan R, et al (2019) Cancer in Africa: the way forward Ecancermedicalscience 13953 PMID: 31552126 PMCID: 6722114

9. Bollyky J (2020) The Growing Cancer Threat in Africa

10. Stefan DC (2015) Cancer care in Africa: an overview of resources J Glob Oncol 1(1) 30-36 https://doi.org/10.1200/JG0.2015.000406 PMID: 28804769 PMCID: 5551648

11. UICC (2019) Cancer Control in Africa: Paving the Way to Universal Health Coverage

12. World Health Organization (2020) Ethiopia Globocan (Geneva: World Health Organization)

13. Federal Ministry of Health (2015) National Cancer Control Plan 2016-2020 (Ethiopia: Federal Ministry of Health)

14. Amare T and Getinet W (2020) Alcohol use and associated factors among high school, college and university students in Ethiopia, systematic review, and meta-analysis, $2018 \mathrm{~J}$ Ment Health 29(4) 455-463 https://doi.org/10.1080/09638237.2019.1677871

15. Ali T and Worku T (2020) Current alcohol consumption and associated factors among school adolescents and youths in Ethiopia: a systematic review and meta-analysis SAGE Open Med 8 2050312120974154 https://doi.org/10.1177/2050312120974154 PMID: 33282302 PMCID: 7682217

16. Deressa Guracho Y, Addis GS, and Tafere SM, et al (2020) Prevalence and factors associated with current cigarette smoking among Ethiopian University students: a systematic review and meta-analysis J Addict 2020 9483164 https://doi.org/10.1155/2020/9483164 PMID: 32373383 PMCID: 7191364

17. Alemu WG, Zeleke TA, and Takele WW, et al (2020) Prevalence and risk factors for khat use among youth students in Ethiopia: systematic review and meta-analysis, 2018 Ann Gen Psychiatry 1916 https://doi.org/10.1186/s12991-020-00265-8 PMID: 32165908 PMCID: 7061479

18. Wubetu AD, Getachew S, and Negash W (2020) Substances use and its association with socio-demographic, family, and environmentrelated factors among technical and vocational education and training college students in Ataye, Ethiopia; an institution-based crosssectional study BMC Public Health 20(1) 1691 https://doi.org/10.1186/s12889-020-09797-w PMID: 33176773 PMCID: 7659098

19. Haileselassie W, Mulugeta T, Tigeneh W, et al (2019) The situation of cancer treatment in Ethiopia: challenges and opportunities $J$ Cancer Prev 24(1) 33-42 https://doi.org/10.15430/JCP.2019.24.1.33 PMID: 30993093 PMCID: 6453587 
20. International aAgency for Research on Cancer and World Health Organization (2018) Globocan Global Cancer Observatory (France: International Agency for Research on Cancer, Geneva: World Health Organization)

21. Abeje S, Seme A, and Tibelt A (2019) Factors associated with breast cancer screening awareness and practices of women in Addis Ababa, Ethiopia BMC Women's Health 19(1) 4 https://doi.org/10.1186/s12905-018-0695-9 PMID: 30616640 PMCID: 6323829

22. Getachew S, Getachew E, and Gizaw M, et al (2019) Cervical cancer screening knowledge and barriers among women in Addis Ababa, Ethiopia PLoS One 14(5) e0216522 https://doi.org/10.1371/journal.pone.0216522 PMID: 31075122 PMCID: 6510425

23. Dibisa TM, Gelano TF, and Negesa L, et al (2019) Breast cancer screening practice and its associated factors among women in Kersa District, Eastern Ethiopia Pan Afr Med J 33144 https://doi.org/10.11604/pamj.2019.33.144.18062 PMID: 31565116 PMCID: 6756780

24. Hussien FM, Hassen AM, and Asfaw ZA, et al (2021) The effect of mobile text messages on knowledge and perception towards cancer and behavioral risks among college students, Northeast Ethiopia: a randomized controlled trial protocol PLoS One 16(7) e0253839 https://doi.org/10.1371/journal.pone.0253839 PMID: 34242256 PMCID: 8270214

25. Aldohaian Al, Alshammari SA, Arafah DM (2019) Using the health belief model to assess beliefs and behaviors regarding cervical cancer screening among Saudi women: a cross-sectional observational study BMC Women's Health 19(1) 6 https://doi.org/10.1186/s12905018-0701-2 PMID: 30621680 PMCID: 6323726

26. Aweke YH, Ayanto SY, Ersado TL (2017) Knowledge, attitude and practice for cervical cancer prevention and control among women of childbearing age in Hossana Town, Hadiya zone, Southern Ethiopia: community-based cross-sectional study PLoS One 12(7) e0181415 https://doi.org/10.1371/journal.pone.0181415 PMID: 28742851 PMCID: 5526548

27. Dehdari T, Dehdari L, Jazayeri S (2016) Diet-related stomach cancer behavior among Iranian college students: a text messaging intervention Asian Pac J Cancer Prev 17(12) 5165

28. Babor TF, Higgins-Biddle JC, and Saunders JB, et al (2011) The Alcohol Use Disorders Identification Test Guidelines for Use in Primary Care (Geneva: World Health Organization)

29. Baker S, Braver E, and Chen L, et al (2002) Drinking histories of fatally injured drivers Inj Prev 8(3) 221-226 https://doi.org/10.1136/ ip.8.3.221 PMID: 12226120 PMCID: 1730866

30. CDC (2017) National Heath Interview Survey

31. Kaleta D, Makowiec-Dąbrowska T, and Dziankowska-Zaborszczyk E, et al (2012) Determinants of heavy smoking: results from the global adult tobacco survey in Poland (2009-2010) Int J Occup Med Environ Health 25(1) 66-79 PMID: 22219059

32. National Cancer Institute (2020) Diet History Questionnaire III (DHQ III) (National Cancer Institute)

33. Stein C and Colditz G (2004) Modifiable risk factors for cancer Brit J Cancer 90(2) 299-303 https://doi.org/10.1038/sj.bjc.6601509 PMID: 14735167 PMCID: 2410150

34. Merten JW, Parker A, and Williams A, et al (2017) Cancer risk factor knowledge among young adults J Cancer Educ 32(4) 865-870 https://doi.org/10.1007/s13187-016-1093-3

35. Abu Samah A, Ahmadian M, and Latiff LA (2015) Insufficient knowledge of breast cancer risk factors among Malaysian female university students Global J Health Sci 8(1) 277-285 https://doi.org/10.5539/gjhs.v8n1p277

36. Imran M, Sayedalamin Z, and Alsulami SS, et al (2016) Knowledge and awareness of colorectal cancer among undergraduate students at King Abdulaziz University, Jeddah, Saudi Arabia: a survey-based study Asian Pac J Cancer Prev 17(5) 2479-2483 PMID: 27268617

37. Sanderson SC, Waller J, and Jarvis MJ, et al (2009) Awareness of lifestyle risk factors for cancer and heart disease among adults in the UK Patient Educ Couns 74(2) 221-227 https://doi.org/10.1016/j.pec.2008.08.003 
38. Ryan AM, Cushen S, and Schellekens H, et al (2015) Poor awareness of risk factors for cancer in Irish adults: results of a large survey and review of the literature Oncologist 20(4) 372-378 https://doi.org/10.1634/theoncologist.2014-0453 PMID: 25746344 PMCID: 4391772

39. Block G, Patterson B, and Subar A (1992) Fruit, vegetables, and cancer prevention: A review of the epidemiological evidence Nutr Cancer 18(1) 1-29 https://doi.org/10.1080/01635589209514201 PMID: 1408943

40. Terry P, Terry JB, and Wolk A (2001) Fruit and vegetable consumption in the prevention of cancer: an update J Intern Med 250(4) 280290 https://doi.org/10.1046/j.1365-2796.2001.00886.x PMID: 11576316

41. Coups EJ and Ostroff JS (2005) A population-based estimate of the prevalence of behavioral risk factors among adult cancer survivors and noncancer controls Prev Med 40(6) 702-711 https://doi.org/10.1016/j.ypmed.2004.09.011 PMID: 15850868

42. McTiernan A (2003) Behavioral risk factors in breast cancer: can risk be modified? Oncologist 8(4) 326-334 https://doi.org/10.1634/ theoncologist.8-4-326 PMID: 12897329

43. Hirvonen K, Wolle A, and Minten B (2018) Affordability of fruits and vegetables in Ethiopia (International Food Policy Research Institute)

44. Gelibo T, Amenu K, and Taddele T, et al (2017) Low fruit and vegetable intake and its associated factors in Ethiopia: a community based cross sectional NCD steps survey Ethiop J Health Dev 31(1) 355-361

45. Trübswasser U, Baye K, and Holdsworth M, et al (2020) Assessing factors influencing adolescents' dietary behaviours in urban Ethiopia using participatory photography Public Health Nutr 2020 1-9

46. Bachewe FN and Minten B (2019) The rising costs of nutritious foods: the case of Ethiopia Intl Food Policy Res Inst 134

47. Klein WMP, Jacobsen PB, and Helzlsouer KJ (2020) Alcohol and cancer risk: clinical and research implications JAMA 323(1) 23-24 https://doi.org/10.1001/jama.2019.19133

48. Ayano G, Yohannis K, and Abraha M, et al (2019) The epidemiology of alcohol consumption in Ethiopia: a systematic review and metaanalysis Sub Abuse Treat Prev Policy 14(1) 1-16

49. Zewdu S, Hanlon C, and Fekadu A, et al (2019) Treatment gap, help-seeking, stigma and magnitude of alcohol use disorder in rural Ethiopia Sub Abuse Treat Prev Policy 14(1) 1-10

50. Mohammed OY, Tesfahun E, and Ahmed AM, et al (2020) Self-reported physical activity status among adolescents in Debre Birhan town, Ethiopia: cross-sectional study PLoS One 15(2) e0229522 https://doi.org/10.1371/journal.pone.0229522 PMID: 32084239 PMCID: 7034904

51. Mohammed OY, Tesfahun E, and Mohammed A (2021) Barriers and Facilitators of Physical Activity among Adolescents of Debre Berhan City Administration; Qualitative Study (Research Square) 